\title{
EPILEPSIAS PARCIAIS FAMILIARES
}

\author{
ELIANE KOBAYASHI*, FERNANDO CENDES*, SOLANGE C. SOUSA**, \\ ANNA E. SCOTONI*, MARIA IMACULADA CARVALHO*, MARILISA M. GUERREIRO*, \\ CARLOS A. M. GUERREIRO*, ISCIA LOPES-CENDES**
}

\begin{abstract}
RESUMO - Objetivo: Investigação das características clínicas e genéticas das epilepsias parciais familiares nos ambulatórios de epilepsia da UNICAMP. Método: História familiar foi obtida em todos os pacientes em acompanhamento, de outubro de 1997 a dezembro de 1998, e aqueles com história familiar positiva para epilepsia foram investigados em detalhe. Heredograma detalhado foi construído para todas as famílias identificadas e história clínica de todos os indivíduos possivelmente afetados foi obtida. Crises e síndromes epilépticas foram classificadas de acordo com as recomendações da ILAE. Sempre que possível, EEG e ressonância magnética foram realizados. Resultados: História familiar positiva foi identificada em 32 pacientes não relacionados. Um total de 213 indivíduos possivelmente afetados foram identificados, dos quais 161 foram clinicamente avaliados. O número de indivíduos afetados por família variou de dois a 23. Epilepsia de lobo temporal (ELT) foi identificada em 22 famílias (68\%), epilepsia de lobo frontal em uma família (3\%), epilepsia com espículas centro-temporais em cinco famílias (15\%) e outras epilepsias parciais benignas da infância em quatro famílias (12\%). A maioria dos indivíduos afetados nas famílias com ELT (69\%) apresentava características clínicas e/ou de EEG consistentes com ELT típica. Entretanto, a gravidade da epilepsia variou, com $76 \%$ dos pacientes com remissão de crises ou bom controle com medicação, e $24 \%$ com crises refratárias, incluindo 7 pacientes que foram submetidos a tratamento cirúrgico. Nas 10 famílias com outras síndromes epilépticas, identificamos 39 indivíduos possivelmente afetados, sendo 23 avaliados clinicamente. Todos apresentavam bom controle de crises (com ou sem medicação) exceto um paciente com epilepsia frontal. Análise dos heredogramas sugeriu herança autossômica dominante com penetrância incompleta em todas as famílias estudadas. Conclusão: A ocorrência familiar é comum nas epilepsias parciais, tanto em adultos como em crianças. A maior parte dos casos estudados foi de pacientes com ELT e a expressão clínica não foi diferente da observada em casos esporádicos, predominando pacientes com bom controle de crises, apesar do caráter heterogêneo. A identificação dos genes envolvidos nos casos estudados poderá ser útil na classificação das síndromes epilépticas, na determinação do prognóstico e regime terapêutico mais indicado.
\end{abstract}

PALAVRAS-CHAVE: epilepsia parcial, genética, recorrência familiar, epilepsia do lobo temporal.

\section{Familial partial epilepsies}

ABSTRACT - Objective: To investigate the clinical and genetic characteristics of familial partial epilepsies. Method: Family history of seizures was questioned in all patients followed in our epilepsy clinics, from October 1997 to December 1998. Those with positive family history were further investigated and detailed pedigrees were obtained. All possibly affected individuals available underwent clinical evaluation. Seizures and epilepsy syndromes were classified according to the ILAE recommendations. Whenever possible, EEG and MRI were performed. Results: Positive family history was identified in 32 unrelated patients. A total of 213 possibly affected individuals were identified, 161 of whom have been evaluated. The number of affected subjects per family ranged from two to 23 . Temporal lobe epilepsy (TLE) was identified in 22 families (68\%), frontal lobe epilepsy in one family (3\%), partial epilepsy with centrotemporal spikes in five families $(15 \%)$, and other benign partial epilepsies of childhood in four families (12\%). Most of the affected individuals in the TLE families (69\%) had

Departamentos de Neurologia*e Genética Médica**, Faculdade de Ciências Médicas, Universidade Estadual de Campinas (UNICAMP), Campinas, SP - Brasil. Aceite: 23-maio-2000.

Dra. Iscia Lopes-Cendes - Departamento de Genética Médica - Faculdade de Ciências Médicas, UNICAMP Caixa Postal 6111 - 13081-970 Campinas SP - Brasil.FAX 19788 8909. E-mail: icendes@unicamp.br 
clinical and/or EEG characteristics of typical TLE. However, the severity of epilepsy was variable, with $76 \%$ of patients with spontaneous seizure remission or good control with medication and $24 \%$ with refractory seizures, including 7 patients that underwent surgical treatment. In the other 10 families, we identified 39 possibly affected subjects, 23 of whom were evaluated. All had good seizure control (with or without medication) except for one patient with frontal lobe epilepsy. Pedigree analysis suggested autosomal dominant inheritance with incomplete penetrance in all families. Conclusion: Family history of seizures is frequent among patients with partial epilepsies. The majority of our families had TLE and its expression was not different from that observed in sporadic cases. The identification of genes involved in partial epilepsies may be usefull in classification of syndromes, to stablish prognosis and optimal treatment.

KEY WORDS: partial epilepsy, genetics, familial recurrence, temporal lobe epilepsy.

A influência de fatores genéticos nas epilepsias sempre foi suspeitada, sendo que a recorrência familiar foi inicialmente demostrada nas epilepsias generalizadas ${ }^{1-5}$ e mais recentemente também nas epilepsias parciais ${ }^{6-11}$. Clinicamente, muitas destas síndromes se apresentam de forma heterogênea, o que pode dificultar o reconhecimento da recorrência familiar. Nas epilepsias parciais com apresentação benigna, muitos pacientes podem não ser corretamente diagnosticados ou simplesmente não ser do conhecimento do restante da família a ocorrência remota de algum episódio compatível com crise epiléptica.

Vários estudos de genética molecular estão em andamento, visando à localização do defeito específico nas várias síndromes. Até o momento pelo menos 18 loci já foram mapeados nas epilepsias idiopáticas. Quatro genes foram identificados, sendo todos relacionados a subunidades de canais iônicos ${ }^{12-15}$. A identificação de um maior número de famílias pode contribuir para que estes estudos esclareçam as bases moleculares das epilepsias e permitam não somente melhor correlação clínica como o desenvolvimento de novas perspectivas terapêuticas.

Os objetivos deste estudo são: estimar a ocorrência de epilepsias parciais familiares em nosso meio e descrever as características clínicas e genéticas dos casos familiares identificados.

\section{MÉTODO}

História familiar foi sistematicamente pesquisada em todos os pacientes acompanhados nos ambulatórios de epilepsia do Hospital das Clínicas da UNICAMP (HC-UNICAMP) a partir de outubro de 1997. Aqueles com história positiva foram posteriormente investigados.

História clínica detalhada e heredograma foram obtidos para todas as famílias identificadas. Crises e síndromes epilépticas foram classificadas de acordo com as recomendações da International League Against Epilepsy (ILAE) ${ }^{16}$.

Foram classificadas como epilepsias parciais aquelas em que a semiologia das crises claramente pudesse ser identificada como crise parcial, com ou sem generalização secundária, em pelo menos um indivíduo. EEG normal ou com atividade epileptiforme generalizada não excluiu o diagnóstico de epilepsia parcial desde que as crises pudessem ser claramente identificadas como crises parciais. Conforme a manifestação clínica, foram subdivididas em: epilepsia de lobo temporal (ELT), epilepsia de lobo frontal (ELF) e epilepsias parciais benignas da infância (EPBI). As EPBI foram assim classificadas, conforme os critérios de Aicardi ${ }^{17}$.

Sempre que possível, registro do eletrencefalograma (EEG) e ressonância magnética (RM) foram realizados, com ênfase nos pacientes com ELT e com controle insatisfatório de crises. Todos os pacientes foram devidamente informados dos objetivos da pesquisa e consentimento pós-informação foi obtido.

\section{RESULTADOS}

Foram identificadas 32 famílias não relacionadas (Tabela 1) com pelo menos dois indivíduos com crises epilépticas compatíveis com epilepsia parcial, num total de 210 indivíduos possivelmente afetados. Desses, avaliamos clinicamente 161 indivíduos.

Epilepsia de lobo temporal (ELT) foi a síndrome epiléptica mais frequentemente encontrada (Tabela 2), num total de 22 famílias e 121 indivíduos possivelmente afetados (dois a 22 por família, 
Tabela 1. 32 famílias com epilepsia parcial.

$\begin{array}{cccc}\begin{array}{c}\text { Epilepsia de } \\ \text { lobo frontal }\end{array} & \begin{array}{c}\text { Epilepsia de } \\ \text { lobo temporal }\end{array} & \begin{array}{c}\text { Epilepsia com } \\ \text { espículas centro } \\ \text { temporais }\end{array} & \begin{array}{c}\text { Outras epilepsias } \\ \text { parciais benignas }\end{array} \\ & & \end{array}$

\begin{tabular}{llccc}
\hline $\mathrm{N}^{\circ}$ de famílias & 1 & 22 & 5 & 4 \\
$\mathrm{~N}^{\circ}$ de pacientes avaliados & 4 & 98 & 11 & 7 \\
$\mathrm{~N}^{\circ}$ de pacientes com crises refratárias & 1 & 16 & 0 & 0 \\
\hline
\end{tabular}

Tabela 2. Características clínicas em 22 famílias com ELT (98 indivíduos avaliados).

\begin{tabular}{lccccc}
\hline ELT com remissão & $\begin{array}{c}\text { ELT com bom } \\
\text { controle medicamentoso }\end{array}$ & ELT refratária & Apenas CTCG & Apenas CF & Crise única \\
\hline $15(22 \%)$ & $37(54 \%)$ & $16(24 \%)$ & $11(11 \%)$ & $11(11 \%)$ & $\begin{array}{c}8(8 \%): \\
5 \text { parcial } \\
3 \text { CTCG }\end{array}$ \\
\hline
\end{tabular}

ELT, epilepsia de lobo temporal; CTCG, crises tônico-clônicas generalizadas; CF, convulsão febril.

média de cinco indivíduos por família). Destes, 98 foram avaliados, sendo 46 homens e 52 mulheres. A idade de início de crises variou de três meses a 35 anos (média= nove anos). Sessenta e oito pacientes apresentavam quadro clínico típico de ELT, com diferentes graus de gravidade $(22 \% \mathrm{com}$ remissão de crises há pelo menos dois anos, $54 \%$ com bom controle medicamentoso e $24 \%$ com crises refratárias). Sete pacientes necessitaram de tratamento cirúrgico. Onze pacientes tiveram apenas crises tônico-clônicas generalizadas (CTCG) que não puderam ter um início parcial identificado, todos com remissão de crises há vários anos. Onze pacientes apresentaram apenas convulsões febris (CF): recorrentes em cinco pacientes e única em sete pacientes. Oito pacientes apresentaram uma única crise (parcial em cinco e CTCG em três pacientes).

Entre os 68 pacientes com ELT, 57 (85\%) apresentaram crises parciais simples (CPS), mais frequentemente do tipo autonômica. Crise parcial complexa (CPC) foi referida por 62 (92\%) pacientes e CTCG secundária ocorreu em 57 (85\%), principalmente no início da epilepsia.

Sessenta e quatro pacientes realizaram EEG, que evidenciou alteração epileptiforme em regiões temporais em 27 indivíduos (42\%) (Fig 1A). Sete pacientes com crises refratárias foram submetidos à monitorização vídeo-EEG, todos com registro de crises nas regiões temporais (Fig 1B). RM foi realizada em 84 pacientes, sendo identificada alteração hipocampal em 49 pacientes (58\%): 45 com atrofia hipocampal (AH) (Fig 2) e quatro com alteração de eixo ou formato do hipocampo.

Epilepsia frontal (ELF) foi identificada em uma família, com sete indivíduos possivelmente afetados, dos quais quatro foram avaliados (idade atual de 14 a 55 anos, média 34). A idade de início das crises variou de sete a 44 anos (média 21 anos). As características semiológicas das crises foram compatíveis com CP em dois indivíduos e CTCG sem início parcial identificável em dois indivíduos. Todos, exceto um paciente, apresentaram evolução benigna, com remissão de crises em um paciente e crise única no outro. EEG foi realizado em dois pacientes, normal em um deles e com atividade epileptiforme em um paciente. Este paciente também foi submetido a monitorização vídeo-EEG, sendo registradas crises de início frontal bilateral com predomínio à direita (Fig 1C). Este paciente apresenta RM normal à análise visual.

Epilepsia parcial benigna da infância (EPBI) foi identificada em nove famílias. - Epilepsia com espículas centro-temporais (EECT) ocorreu em cinco famílias, com 16 indivíduos possivelmente 


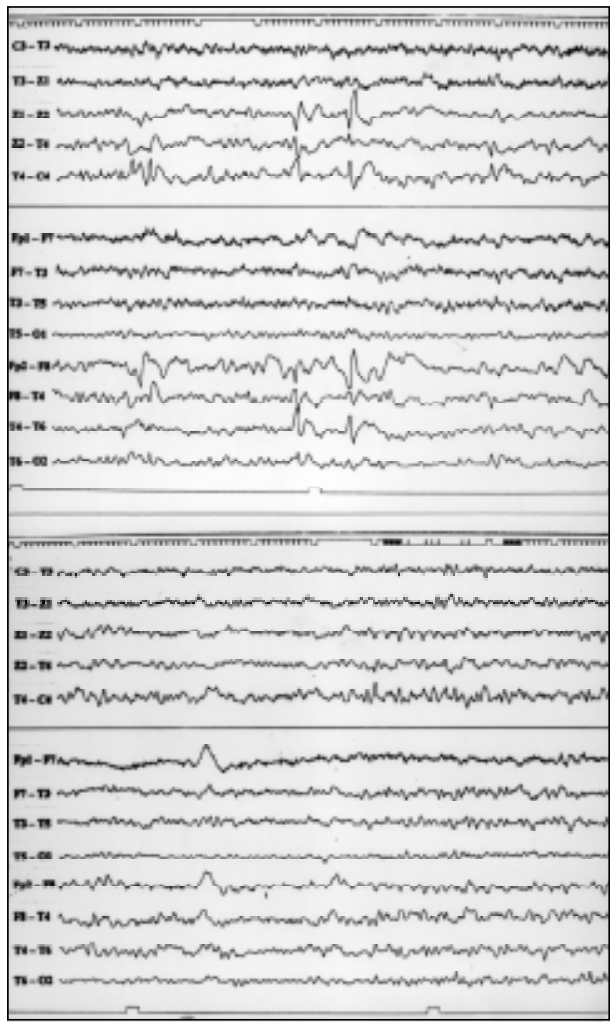

Fig 1A. EEG interictal de paciente com ELT e crises refratárias e RM com atrofia hipocampal direita. $O$ traçado mostra espículas, ondas agudas e ondas lentas intermitentes na região temporal anterior e mediobasal direita. afetados (máximo de seis indivíduos por família). Destes, $11(69 \%)$ foram avaliados (oito homens e três mulheres) com idade variando entre oito e 67 anos (média de 25 anos). O início das crises ocorreu entre três e 16 anos (média= nove anos) e todos apresentaram boa evolução, com crises controladas ou em remissão há pelo menos dois anos. Seis indivíduos realizaram EEG, sendo normal em cinco e com espículas centrotemporais em um indivíduo. Seis pacientes realizaram TC de crânio, sendo todas normais.

- Epilepsia parcial benigna da infância com paroxismos occipitais (EPPO) foi identificada em duas famílias, com oito indivíduos possivelmente afetados. Três pacientes foram avaliados, com idade média atual de 20 anos (variando de sete a 38 anos). Idade de início das crise foi de dois a cinco anos. Apenas um paciente não apresentou sintomas visuais no início das crises. Dois pacientes realizaram EEG e apenas um mostrou atividade epileptiforme.

- Epilepsia parcial benigna da infância que não pôde ser classificada nos grupos anteriores foi identificada em duas famílias. Os tipos de crises identificados foram: sensitiva em dois pacientes, motora em um paciente e visual em quatro pacientes. Um paciente teve uma única crise febril e um paciente teve uma única CTCG sem início parcial identificável. A evolução foi benigna em todos os pacientes, sendo que a maioria deles apresentou apenas dois ou três episódios durante a vida.

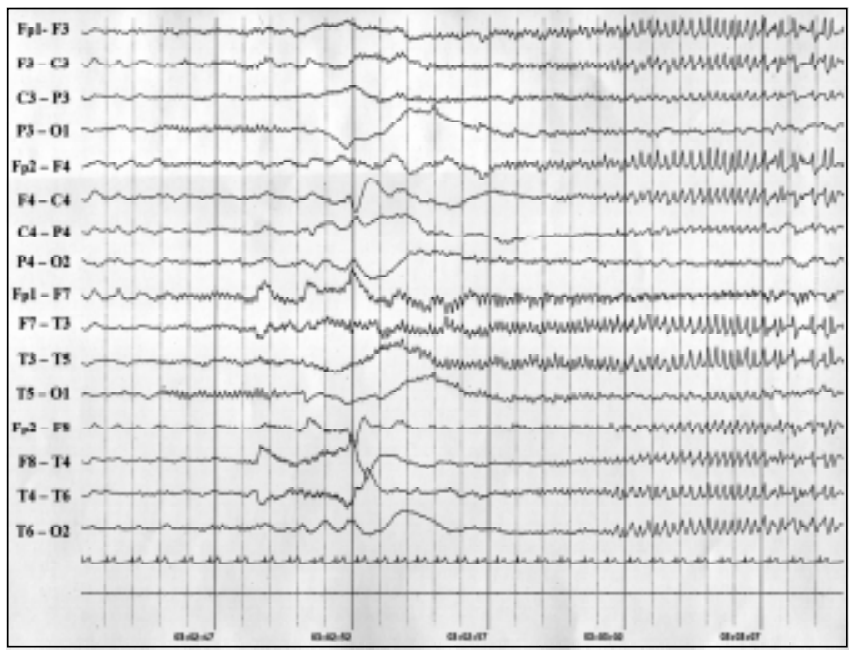

Fig 1B. EEG ictal de paciente com ELT crises refratárias, com início eletrográfico na região temporal esquerda. Apresentava atrofia hipocampal esquerda à RM e foi submetido a ressecção temporal envolvendo amígdala e hipocampo (classe IIa de Engel após 25 meses de seguimento). 


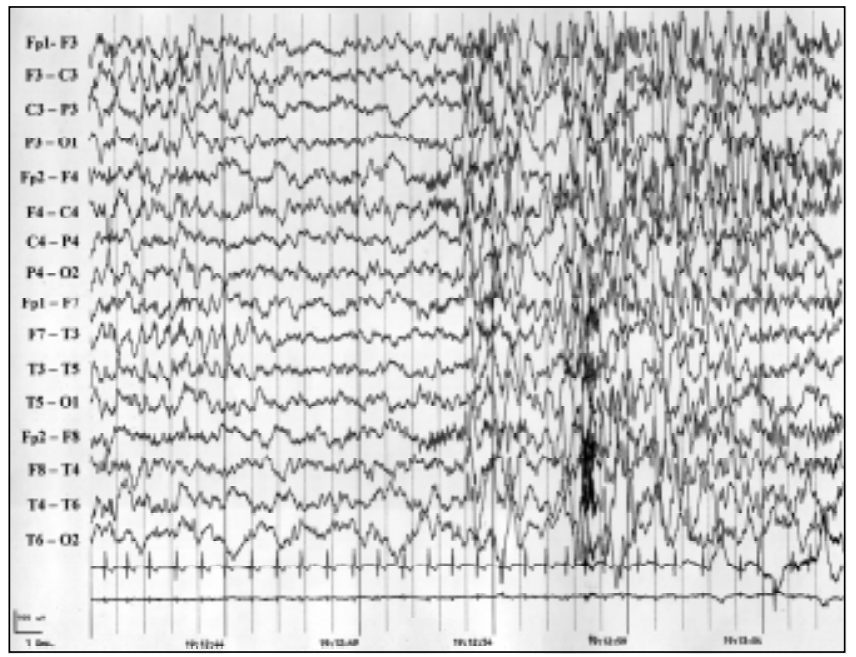

Fig 1C. Registro ictal de crise de início bifrontal com predomínio à direita (reversão de fase em $\mathrm{F} 4$ ) no único paciente com quadro refratário de epilepsia de lobo frontal (RM normal à análise visual).

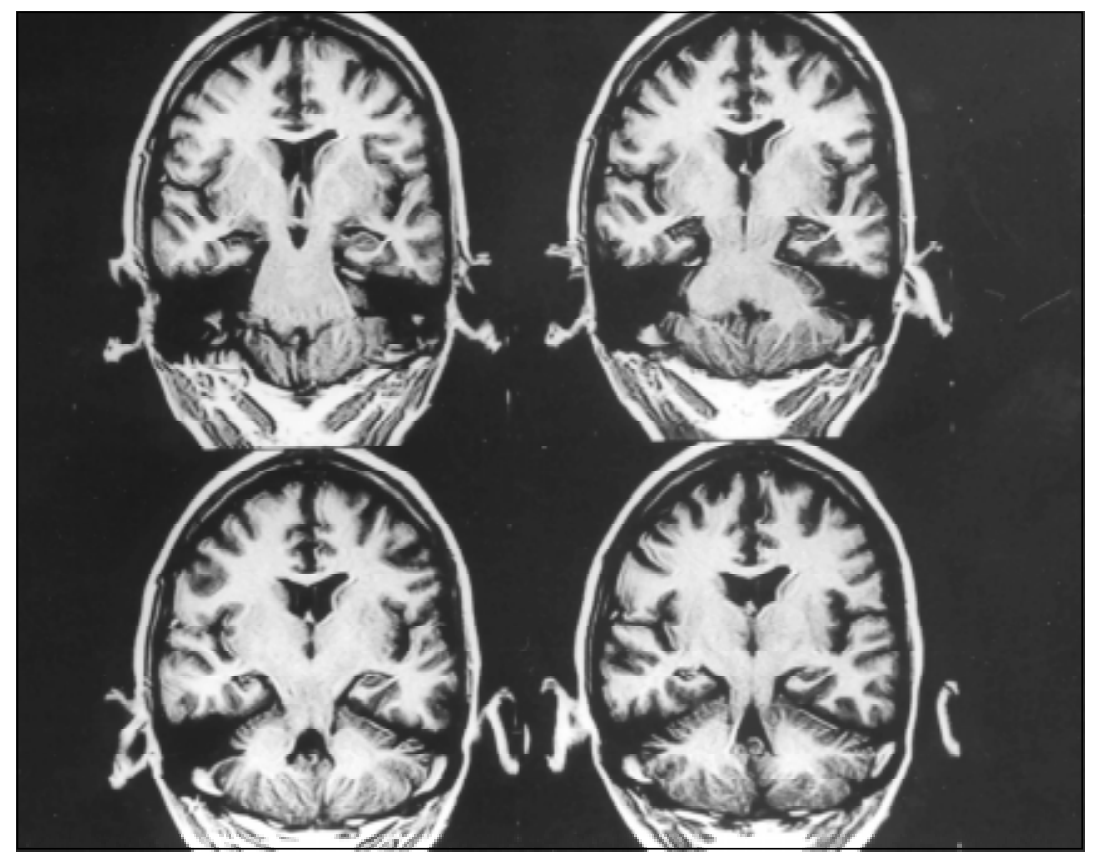

Fig 2. RM de paciente com ELT e crises refratárias (irmã da paciente cujo EEG foi ilustrado na figura 1A), em cortes coronais Inversion Recovery, mostrando atrofia hipocampal direita.

Análise dos heredogramas sugeriu padrão de herança autossômica dominante com penetrância incompleta em todas as famílias identificadas (Fig 3). É interessante notar a grande frequência de história familiar de epilepsia presente em ambos os lados paterno e materno de várias famílias. 


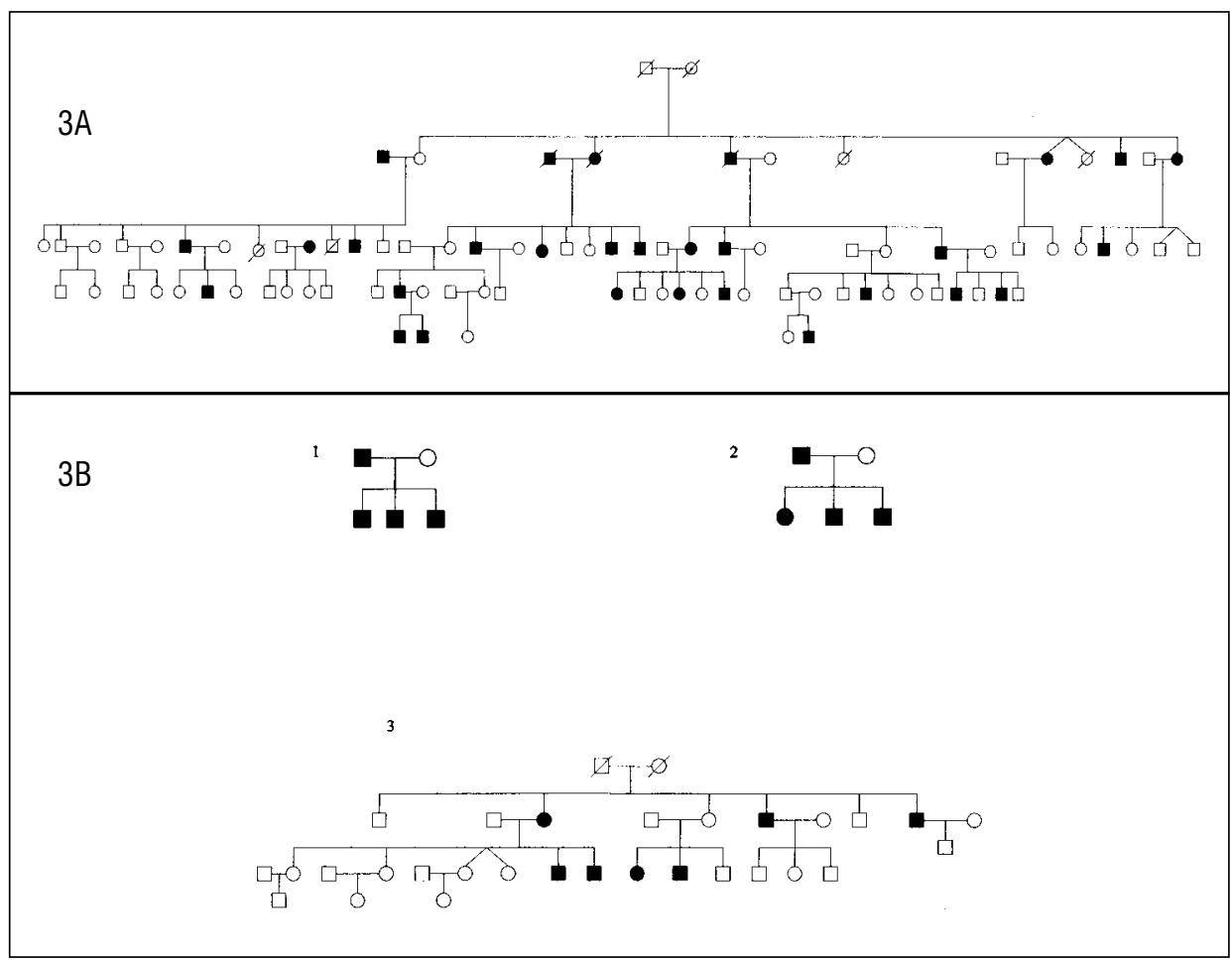

Fig 3. Heredogramas de quatro famílias com epilepsia parcial: 3A, família com ELT; 3B, famílias com epilepsia parcial benigna com espículas centrotemporais. (1), epilepsia parcial benigna da infância (2) e epilepsia frontal (3), $\square$ - Indivíduo com pelo menos uma crise febril ou afebril; $\square \bigcirc$ Indivíduo não afetado; $\square \varnothing$ Indivíduo falecido.

\section{DISCUSSÃO}

Nosso grupo de pacientes foi constituído em sua maior parte por famílias com ELT. Não podemos descartar a possibilidade de um viés na população estudada, uma vez que os pacientes que frequentam os ambulatórios de epilepsia do HC-UNICAMP são encaminhados de outros serviços de atendimento, primários e secundários. Portanto, muitos casos de epilepsia benigna e de fácil controle não são rotineiramente avaliados pela nossa equipe.

Entretanto, mesmo em nossa amostragem "selecionada", conseguimos identificar casos familiares de epilepsias parciais benignas. Muitos pacientes não sabiam relatar num primeiro questionamento a ocorrência de crises em seus familiares, em geral pela extrema benignidade dos episódios.

A única família identificada com epilepsia frontal apresenta um único indivíduo com crises refratárias noturnas, compatível com quadro de epilepsia frontal familiar previamente descrito ${ }^{7}$. Dentre os pacientes com ELT, pudemos observar heterogeneidade importante intra e interfamiliar, semelhante a observações de outra série previamente publicada ${ }^{10}$.

Acredita-se que a real prevalência das epilepsias parciais familiares seja subestimada, principalmente devido à dificuldade de obtenção de informação relativa aos antecedentes familiares e de seleção e classificação dos indivíduos afetados. Interrogatório sistemático e ativo em todos os pacientes avaliados pode auxiliar na identificação de um número maior de famílias, permitindo um estudo clínico mais detalhado em nosso meio. Além disso, o estudo da população atendida em 
serviços primários e secundários de saúde, principalmente na faixa pediátrica, pode fornecer dados epidemiológicos mais representativos.

A importância do reconhecimento e estudo das epilepsias parciais familiares está relacionada à perspectiva de se aprofundar nos mecanismos moleculares provavelmente envolvidos na epileptogênese que possibilitarão o desenvolvimento de novas estratégias terapêuticas no futuro.

\section{REFERÊNCIAS}

1. Ottmann R, Lee JH, Risch N, et al. Clinical indicators of genetic susceptibility to epilepsy. Epilepsia 1996;37:353-361.

2. Hauser WA, Anderson VE. Genetics of epilepsy. In Pedley TA, Meldrum BS. (eds). Recent advances in epilepsy 3. Edinburgh: Churchill Livingstone,1986:21-36.

3. Andermann E. Multifactorial inheritance of generalized and focal epilepsy. In: Anderson VE, Hauser WA, Penry JK, Sing CF (eds). Genetic basis of the epilepsies. New York: Raven Press, 1982:355-374.

4. Anneger JF, Hauser WA, Anderson VE. Risk of seizures among relatives of patients with epilepsy: families in a defined population. In Anderson VE, Hauser WA, Sing L, Porter R (eds) The genetic basis of the epilepsies. New York: Raven Press, 1982:151-159.

5. Berkovic SF, Howell RA, Hay DA, Hopper JL. Epilepsies in twins: genetics of the major epilepsy syndromes. Ann Neurol 1998;43:435-445.

6. Ottman R. Genetics of the partial epilepsies: a review. Epilepsia 1989;30:107-111.

7. Phillips HA, Scheffer IE, Berkovic SF, et al. Localization of a gene for autosomal dominant nocturnal frontal lobe epilepsy to chromosome 20q13.2. Nat Genet 1995;10:5117-5118.

8. Ottman R, Risch N, Hauser WA, et al. Localization of a gene for partial epilepsy to chromosome 10q. Nat Genet 1995;10: 56-60.

9. Scheffer IE, Phillips HA, O'Brien CE, et al. Familial partial epilepsy with variable foci: a new partial epilepsy syndrome with suggestion of linkage to chromosome 2. Ann Neurol 1998;44:890-899.

10. Cendes F, Lopes-Cendes I, Andermann E, Andermann F. Familial temporal lobe epilepsy: a clinically heterogeneous syndrome. Neurology 1998;50:554-557.

11. Berkovic SF, Scheffer IE. Genetics of the epilepsies. Curr Opin Neurol 1999;12:177-182.

12. Bievert C, Schoeder BC, Kubisch C, Berkovic SF, Propping P, Jentsch TJ, Steinlein OK. A potassium channel mutation in neonatal human epilepsy. Science 1998;279:403-406.

13. Singh NA, Charlier C, Stauffer D, et al. A novel potassium channel gene, KCNQ2, is mutated in an inherited epilepsy of newborns. Nat Genet 1998;18:25-29.

14. Steinlein OK, Mulley JC, Propping P, et al. A missense mutation in the neuronal nicotinic acetylcholine receptor a4 subunit is associated with autosomal dominant nocturnal frontal lobe epilepsy. Nature Genet 1995;11:201-203.

15. Wallace RH, Wang DW, Sing R, et al. Febrile seizures and generalized epilepsy associated with a mutation in the Na+channel b1 subunit gene SCN1B. Nat Genet 1998;19:366-370.

16. Comission on Classification and Terminology of the International League Against Epilepsy. Proposal for a revised classification of epilepsies and epileptic syndromes. Epilepsia 1989;30:389-399.

17. Aicardi J. Epileptic syndromes in childhood. Epilepsia 1988;29(Suppl3): S1-S5. 